\title{
Adhesion Molecule GD18 on Polymorphonuclear Cells Correlates to the Lung Injury Caused by Continuous Infusion of Endotoxin in Sheep
}

\author{
Hiroshi Kubo, Tatsuo Tanita, Kaoru Koike*, \\ Sadafumi Ono and Sigefumi Fujimura \\ Department of Thoracic Surgery, Institute of Development, \\ Aging and Cancer, Tohoku University, Sendai 980-77, and \\ * Pulmonary Division, Miyagi Cancer Center, Natori 981- \\ 12
}

Kubo, H., Tanita, T., Koike, K., Ono, S. and Fujimura, S. Adhesion Molecule CD18 on Polymorphonuclear Cells Correlates to the Lung Injury Caused by Continuous Infusion of Endotoxin in Sheep. Tohoku J. Exp. Med., 1995, 177 (3), 213-222 — We investigated the mechanisms of increase in the pulmonary vascular permeability, focusing on the changes in the peripheral white blood cell (WBC) counts and the surface expression of CD18 on polymorphonuclear cells (PMNs). Anesthetized sheep with chronic lung lymph fistulas were used in this study. We infused synthetic endotoxin (LPS) at a rate of $10 \mathrm{ng} / \mathrm{kg} / \mathrm{min}$ (i.v.) continuously for $24 \mathrm{hr}$. We measured lung lymph flow, lymph-to-plasma protein concentration ratio and WBC counts in blood and lung lymph, and the PMNs' surface expression of CD18 before and at 2, 10 and $24 \mathrm{hr}$ after the start of endotoxin infusion, respectively. CD18 was analyzed by flow cytometry using monoclonal anti-CD18 antibody. We found that the pulmonary vascular permeability increased during 2-4 $\mathrm{hr}$ after the start of endotoxin infusion, and returned to the baseline over $10 \mathrm{hr}$. At time $2 \mathrm{hr}$ period, the number of WBCs in the lung lymph increased, the number of peripheral WBCs, mostly PMNs, decreased and the surface expression of CD18 on the peripheral PMNs was up-regulated. At time 10 and $24 \mathrm{hr}$, the number of WBCs in lung lymph decreased, the number of peripheral WBCs increased and CD18 expression was down-regulated. These data indicate that up-regulation of CD18 expression promotes the PMN adherence to the pulmonary endothelium, migration into the lung and increases the pulmonary vascular permeability. We conclude that the continuous endotoxin infusion up-regulates CD18, which contributes to the PMN migration into the lung. — adhesion molecules; CD 18; continuous endotoxin infusion; sheep

The essence of adult respiratory distress syndrome (ARDS) is thought to be an increase in permeability of pulmonary vessels resulting in edema. In lung injury, the localized aggregation of polymorphonuclear cells (PMNs) within the pulmonary microvasuculature is an important prerequisite (Powe 1982). In-

Received February 28, 1995; revision accepted for publication September 15, 1995. 
creased PMN adhesiveness to endothelial cells is a critical, early step in the sequence of events leading to PMN-mediated injury. One of the primary mechanisms of PMN adherence depends on adhesion molecule, CD18. A monoclonal antibody against CD18 prevents reperfusion-induced lung injury (Horgan et al. 1990) and PMN accumulation in cutaneous inflammation (Mileski et al. 1992). CD18 influences not only adhesion but degranulation of PMNs (Schleiffenbaum et al. 1989). These studies suggest that CD18 may play an important role in PMN-mediated lung injury. Migration and sequestration of PMNs into the lung during acute lung injury are well investigated. However, the relationship between PMN migration and adhesion molecule CD18 in endotoxemia is still unclear. Since endotoxin is supplied to whole body continuously during endotoxemia, we planned to give continuously endotoxin to sheep for $24 \mathrm{hr}$. To examine this hypothesis we tried to see whether CD18 would contribute to the endotoxin-induced lung injury, and whether surface expression of CD18 would change in endotoxemia and, if so, how the relationship between PMN migration and PMN surface expression of CD18 would be.

\section{Methods}

\section{Lung lymph fistula}

Nine sheep weighing $35-50 \mathrm{~kg}$ were used for this study. Sheep were injected with thiopental sodium (10 mg/kg, i.v.) and anesthesia was maintained with a mixture of $2 \%$ enflurane, $50 \%$ oxygen and air. Through the neck vessels, we introduced a 7-Fr Swan-Ganz thermodilution catheter (93A-131H-7F; Edwards Lab., Irvine, CA, USA) into the pulmonary artery, and an arterial catheter into the aorta. Preparation of the lung lymph fistula was performed using a modification of the method outlined by Staub et al. (1975). A right thoracotomy was performed in the 7th intercostal space, the lung was retracted, and the pleura was opened to expose the caudal mediastinal lymph node (CMN). The caudal portion of the CMN was tied off to remove systemic lymph contribution. The efferent duct was cannulated with silicon catheter (602-155; Dow Corning, Midland, MI, USA). We started the examination 7 days after the operation day.

\section{Endotoxin infusion}

After a 2-hr baseline period, we infused synthetic endotoxin (Lipid A, LA-15-PP(506), Daiichi Pure Chemicals, Tokyo) i.v. at a rate of $10 \mathrm{ng} / \mathrm{kg} / \mathrm{min}$ continuously for $24 \mathrm{hr}$. Mean pulmonary arterial pressure (PAP) was measured continuously with a force transducer (P23-ID; Gould, Santa Ana, CA, USA) leveled at the right atrium. Pulmonary capillary wedge pressure (PCWP) and cardiac output (CO) were measured at 30-min intervals for $24 \mathrm{hr}$. CO was measured by a thermodilution method and the value was determined as the mean of three measurements of $5 \mathrm{ml}$ injections of $5 \%$ glucose at $0^{\circ} \mathrm{C}$ through the venous port of the Swan-Ganz catheter. Pulmonary vascular resistance (PVR) was 
calculated as (PAP-PCWP)/CO.

\section{Lymph-dynamics}

The lymph from CMN was collected in plastic tubes, added heparin of $0.02 \mathrm{ml}$ to prevent clotting, and the weight was measured every $30 \mathrm{~min}$. Total protein concentrations of this lymph and the plasma, collected each $30 \mathrm{~min}$, were measured by the Biuret method using an automated analyzer (AU-500; Olympus, Tokyo). Lymph protein clearance (Clym) was calculated as (lymph flow) $\times$ (lymph to plasma protein concentration ratio). We measured the white blood cell (WBC) counts in lymph using automated analyzer (CC800; Toa Iryodensi, Kobe).

\section{Peripheral white blood cells}

The heparinized blood specimens were collected at every 30 -min interval from the aortic catheter. The WBC counts were measured using an automated analyzer. Differential cell counts were performed on WBCs using Giemsa stained smears. We calculated the percentage of PMNs from counting of 200 WBCs.

\section{Measurement of surface CD18 expression}

The heparinized blood specimens were collected through the aortic catheter before and at 2, 10 and $24 \mathrm{hr}$ after the start of endotoxin infusion, respectively. Blood specimen was cooled on ice rapidly, and the following process of staining was carried out in the dark at $4^{\circ} \mathrm{C}$. A hundred ml of blood was mixed with aggregated IgG (sheep gamma globulin, D612; Rockland, PA, USA) $10 \mu$ for 20 min, then combined with FITC-conjugated anti-CD18 monoclonal antibody (F 839; Dakopatts, Gilbertsville, Denmark) $10 \mu \mathrm{l}$ for $30 \mathrm{~min}$. The blood was washed twice with phosphate buffer solution (PBS), subjected to erythrocyte lysis $\left(\mathrm{NH}_{4} \mathrm{Cl}, \mathrm{KHCO}_{3}\right.$ and EDTA), and washed again, then suspended at the final concentration of $10^{6}$ cells $/ \mathrm{ml}$ in PBS. Negative controls were stained with control antibody (mouse IgG, 31469; Cappel, Durham, NC, USA) and FITCconjugated secondary antibody (4353; Tago Immunologicals, Camarillo, CA, USA) instead of anti-CD18 antibody. Flow cytometry was carried out using a Spectrum III (Becton Deckinson Immunocytometry Systems, San Jose, CA, USA). Fluorescence intensity was determined directory on a linear scale.

\section{Data analysis}

Data are expressed as means \pm s.e. Data were analysed by Student's $t$-test with significance accepted as $p<0.05$.

\section{Results}

\section{Pulmonary hemodynamics}

After the start of endotoxin infusion, PAP increased rapidly, but decreased to the baseline over $2 \mathrm{hr}$. PCWP was stable at $7.6 \pm 0.8(\mathrm{mmHg})$ during the 
experiment. CO decreased at 1-hr period, but went back to the baseline over $2 \mathrm{hr}$. Then PVR increased at 1-hr period, but went back to the baseline over $2 \mathrm{hr}$ (Table 1).

\section{Pulmonary lymph-dynamics}

Lung lymph flow increased at 2-hr period by more than three times, but decreased by degrees and went back to the baseline over $10 \mathrm{hr}$. Lymph to plasma protein concentration ratio decreased at the 1st hour, but increased at the 2nd hour. Then it went back to the baseline over $10 \mathrm{hr}$ (Table 1). Then Clym increased at $2 \mathrm{hr}$ and went back to the baseline over $10 \mathrm{hr}$ (Fig. 1). WBC counts in the lymph increased by eight times, but went back to the baseline over $10 \mathrm{hr}$ (Fig. 2).

TABLE 1. Changes in pulmonary hemodynamics and lymph-dynamics

\begin{tabular}{cccccc}
\hline & PAP $(\mathrm{mmHg})$ & CO (liter/min) & PVR $(\mathrm{mmHg} / \mathrm{liter} / \mathrm{min})$ & $\mathrm{QL}(\mathrm{g} / 30 \mathrm{~min})$ & $\mathrm{L} / \mathrm{P}$ \\
\hline Baseline & $12.8 \pm 0.5$ & $6.11 \pm 1.54$ & $1.01 \pm 0.09$ & $4.11 \pm 0.71$ & $0.70 \pm 0.05$ \\
$1 \mathrm{hr}$ & $25.8 \pm 2.5^{*}$ & $4.73 \pm 1.51^{*}$ & $3.68 \pm 0.05^{*}$ & $6.73 \pm 0.79$ & $0.65 \pm 0.06$ \\
$2 \mathrm{hr}$ & $15.3 \pm 2.0$ & $6.10 \pm 1.42$ & $0.90 \pm 0.24$ & $13.42 \pm 0.73^{*}$ & $0.76 \pm 0.04$ \\
$10 \mathrm{hr}$ & $14.5 \pm 1.8$ & $6.36 \pm 0.96$ & $0.71 \pm 0.03$ & $5.34 \pm 0.47$ & $0.73 \pm 0.04$ \\
$24 \mathrm{hr}$ & $13.8 \pm 0.5$ & $6.18 \pm 1.11$ & $0.90 \pm 0.08$ & $4.81 \pm 0.30$ & $0.75 \pm 0.07$ \\
\hline
\end{tabular}

PAP, mean pulmonary arterial pressure; CO, cardiac output; PVR, pulmonary vascular resistance; $\mathrm{QL}$, lung lymph flow; $\mathrm{L} / \mathrm{P}$, lymph to plasma protein concentration ratio. Values are mean \pm s.e. ${ }^{*} p<0.05$ vs. baseline.

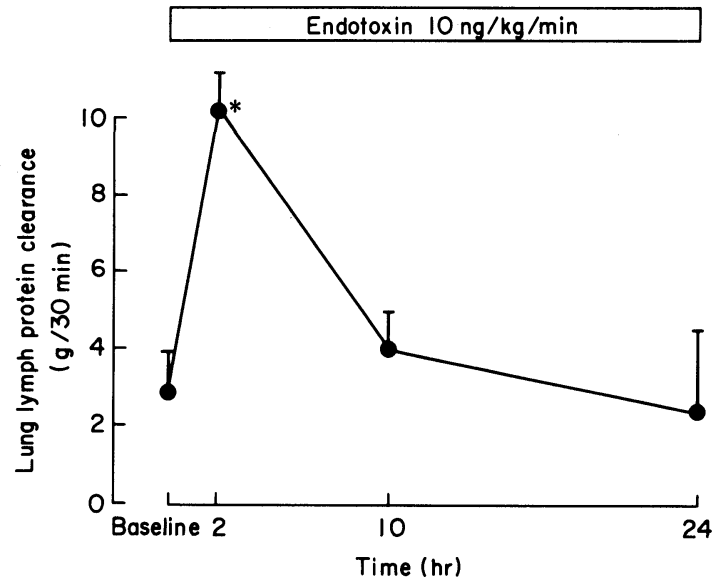

Fig. 1. Changes in the lymph protein clearance. Lymph protein clearance increased at $2 \mathrm{hr}$ after the start of endotoxin infusion, then went back to the Baseline over $10 \mathrm{hr} .{ }^{*} p<0.05$ vs. baseline. 


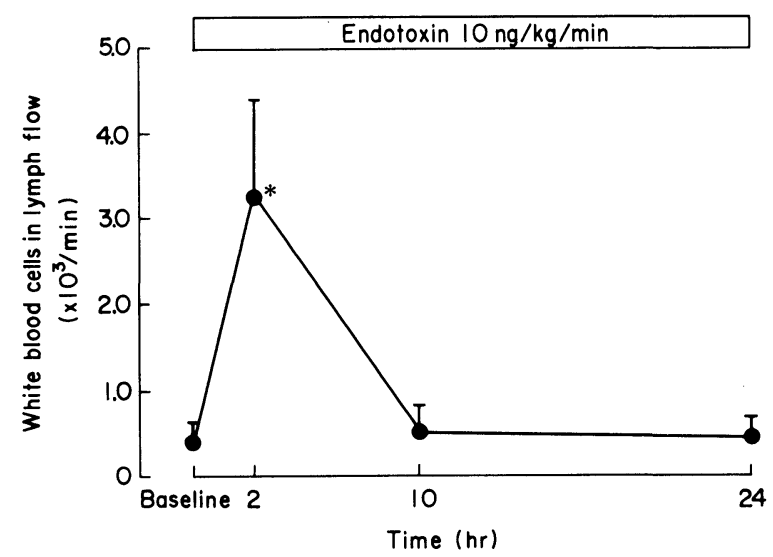

Fig. 2. Changes in white blood cell (WBC) counts in lymph flow. The number of WBCs in lymph flow increased at $2 \mathrm{hr}$ after the start of endotoxin, but went back to the baseline over $10 \mathrm{hr} .{ }^{*} p<0.05$ vs. baseline.

\section{Peripheral blood}

WBC counts decreased rapidly after the start of endotoxin infusion, but increased 2 fold over $10 \mathrm{hr}$ (Fig. 3). The percentage of PMNs fell to $4.4 \pm 0.6(\%)$ at $2 \mathrm{hr}$ from $39.7 \pm 3.4$ at baseline, but increased to $74.0 \pm 3.0$ at $10 \mathrm{hr}$ and $69.7 \pm$ 1.2 at $24 \mathrm{hr}(p<0.05)$.

\section{CD18 expression}

The percentage of CD18 positive PMNs increased at $2 \mathrm{hr}$, but decreased over $10 \mathrm{hr}$ (Fig. 4). The CD18 expression was up-regulated $2 \mathrm{hr}$, but down-regulated

Endotoxin $10 \mathrm{ng} / \mathrm{kg} / \mathrm{min}$

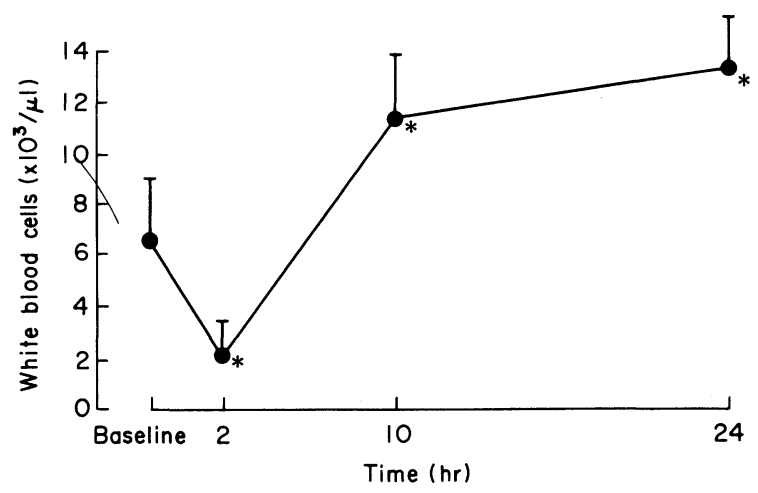

Fig. 3. Changes in peripheral white blood cell counts. The number of peripheral WBCs decreased at $2 \mathrm{hr}$ after the start of endotoxin infusion, and then increased over $10 \mathrm{hr}$ in endotoxemia. ${ }^{*} p<0.05$ vs. baseline. 


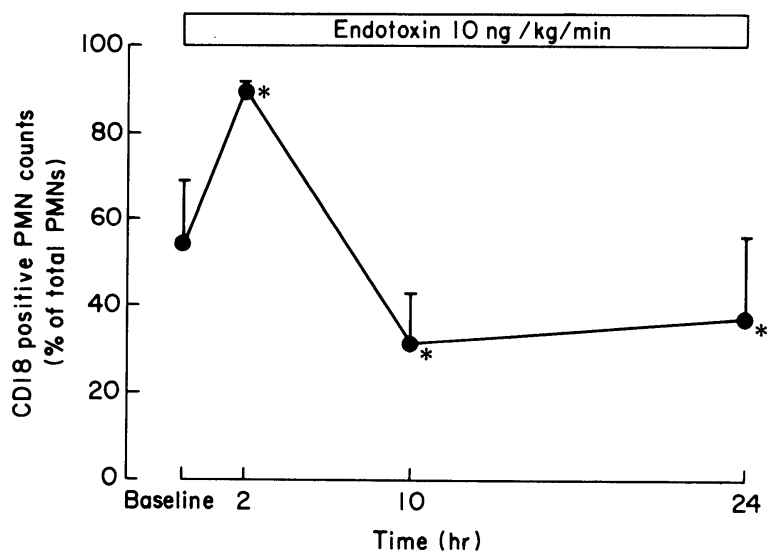

Fig. 4. Changes in the number of CD18 positive polymorphonuclear cells (PMNs). The percentage of CD18 positive PMNs increased at $2 \mathrm{hr}$, but decreased over $10 \mathrm{hr} . \quad{ }^{*} p<0.05$ vs. baseline.

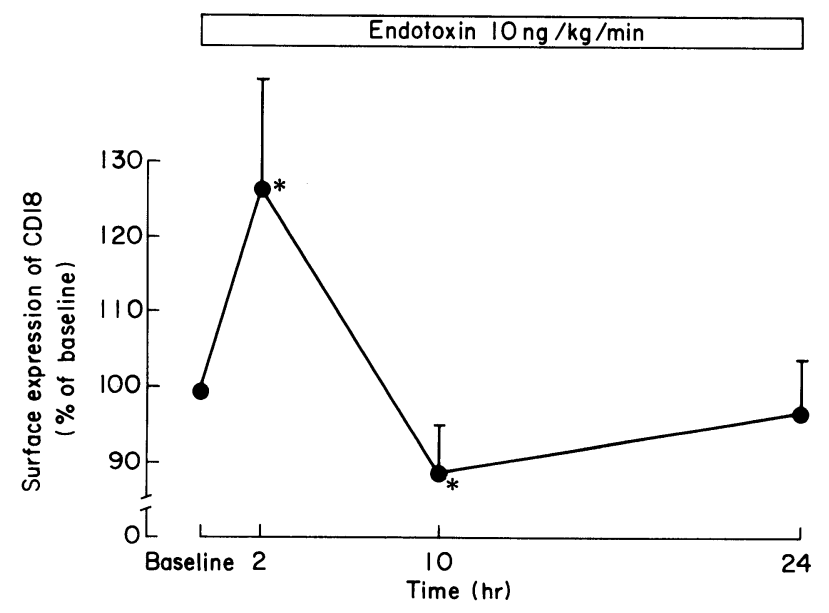

Fig. 5. Changes in surface expression of CD18 on PMNs. CD18 expression was up-regulated at $2 \mathrm{hr}$ after the start of endotoxin infusion, but down-regulated over $10 \mathrm{hr} .{ }^{*} p<0.05$ vs. baseline.

at $10 \mathrm{hr}$ (Fig. 5). Clym and WBC counts in lymph were well correlated with the percentage of CD18 expression (Figs. 6 and 7).

\section{Discussion}

We found that two different changes occurred in $24 \mathrm{hr}$ of continuous endotoxin infusion. So we call the phase up to $2 \mathrm{hr}$ "early phase", and the phase over $10 \mathrm{hr}$ after the start of endotoxin infusion "late phase". The pulmonary lymph flow and lymph to plasma protein concentration ratio ( $\mathrm{L} / \mathrm{P}$ ratio) increased in the early phase. These changes can be interpreted as an increase in pulmonary 


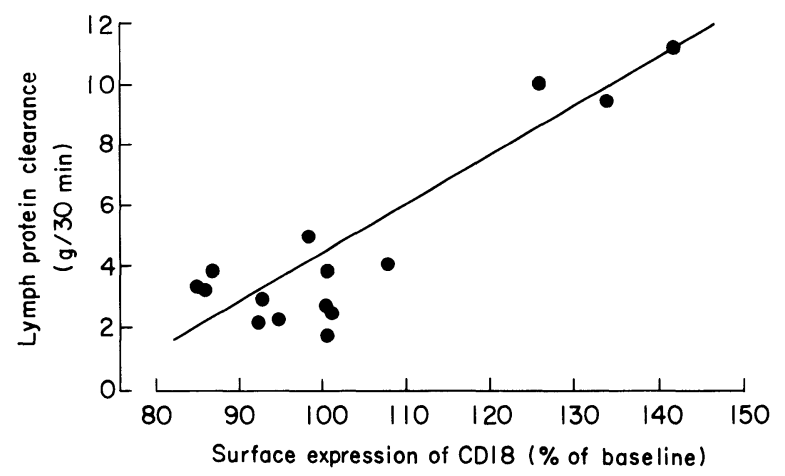

Fig. 6. Correlation of surface expression of CD18 on PMNs and lymph protein clearance (Clym). Clym was well correlated with the percentage of CD18 expression.

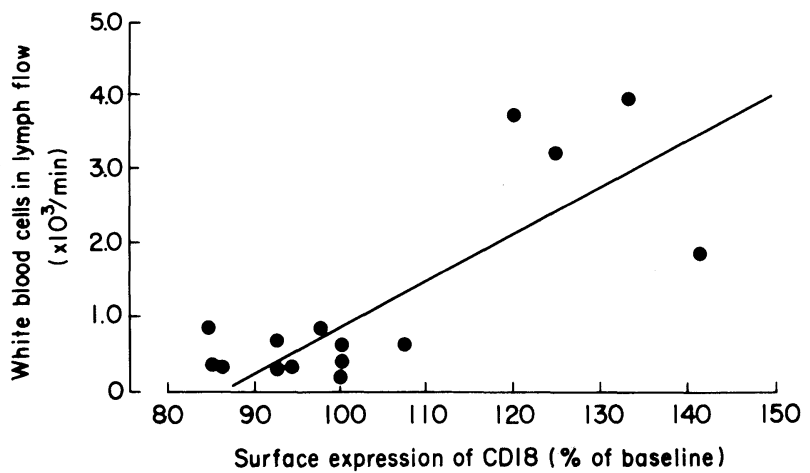

Fig. 7. Correlation of surface expression of CD18 on PMNs and WBC counts in lymph. WBC counts in lymph was well correlated with the percentage of CD18 expression.

vascular permeability. Since, pulmonary lymph flow and L/P ratio went back to the baseline in the late phase, the pulmonary vascular permeability improved in spite of continuous endotoxin infusion.

The responses of number of peripheral WBCs in our early phase, were similar to those already described in sheep injected with endotoxin singly (Warner 1988). The number of WBCs, mostly PMNs, decreased rapidly after the start of endotoxin infusion and remained low through the early phase. The number of circulating neutrophils declined after intravenous endotoxin infusion (Warner 1988), and the neutrophils were shown to be sequestered in the pulmonary vascular bed (Koike et al. 1986). These phenomena interpreted that WBCs migrated into the lung and were sequestered. In this study, WBC counts in lymph increased in the early phase which showed that WBCs were sequestered in the lung and moved into the lymph flow. In contrast, the number of peripheral WBCs in the late phase increased twice in the baseline. These WBCs were 
mostly mature PMNs. Therefore, these cells likely came from storage organs, such as, the bone marrow, lung, spleen, and liver. Since, the lung is one of the largest storehouse (Staub et al. 1982) of PMNs in sheep, it is likely that the most part of this increase in the peripheral WBCs was due to the recruitment from the lung.

PMN-mediated tissue injury requires the adherence of these cells to endothelium (Arfors et al. 1987). Although some investigators indicated that the leukopenia (WBCs migrated into the lung) seen after endotoxin infusion was not CD18 dependent (Lundberg 1990; Thomas et al. 1992), yet anti-CD18 monoclonal antibody brought about a recovery of number of peripheral PMNs faster and prevented an accumulation of PMNs in the lung (Doerschuk 1992). PMNs at sites of inflammation in vivo up-modulate their surface expression of adhesionpromoting CD18 glycoprotein during their recruitment from the circulating, intravascular leukocyte pool (Freyer 1989). In the early phase, CD18-positive PMNs increased and surface expression of CD18 was up-regulated. The quantitative increase in PMNs-surface CD18 provides a mechanism for initiating leukoaggregation and sequestration of PMNs (Arnaout et al. 1985). In our study, WBC counts were correlated with the CD18 expression on peripheral PMNs. Therefore, PMNs were shown to be sequestered in the pulmonary vascular bed and adhered to the endothelium. After adhesion, PMNs may have released harmful oxidative and proteolytic products, namely, oxygen radicals and/or protease. In contrast, CD18-positive PMNs decreased and surface expression of CD18 was downregulated in the late phase. Therefore PMNs-adhesion decreased and PMNs may have been peeled from the endothelium, then harmful products released from PMNs may have been decreased, and endothelial damage may have been improved. Our data suggest that CD18 may contribute to the sequestration of PMNs into the lung, however, not to the trans-endothelial migration (Abbassi et al. 1991).

Despite the continuous infusion of endotoxin, the increase in expression of CD 18 on the surface of PMNs and the increase in lung lymph clearance were transient. One possibility is the tolerance of PMNs against LPS. Continuous or repetitive infusion of LPS decreases productions of cytokines (Virca et al. 1989). Repetitive infusions of low doses of endotoxin markedly reduced TNF activity, and inhibited adhesion of PMNs to nylon fibers (Barroso-Aranda et al. 1991). One of the reasons for this tolerance is thought to be a down-regulation of CD 14 by synthetic endotoxin (LPS) (Labeta et al. 1993). In our study, the continuous infusion of LPS may have decreased the production of cytokines, namely, TNF or IL 8 (Mackensen et al. 1991), and then may have decreased the surface expression of CD 18 and lymph protein clearance. These results indicate that up-regulation of CD18 expression promotes the PMNs adherence to the pulmonary vascular endothelium, and increases the pulmonary vascular permeability. 


\section{Acknowledgement}

We are grateful to Ms. Nitanai, E. for her technical support on flow cytometer.

\section{References}

1) Abbassi, O., Lane, C.L., Krater, S., Kishimoto, T.K., Anderson, D.C., McIntire, L.V. \& Smith, C.W. (1991) Canine neutrophil margination mediated by lectin adhesion molecule-1 in vitro. J. Immunol., 147, 2107-2115.

2) Arfors, K.E., Lundberg, C., Lindbom, L., Lunberg, K., Beatty, P.G. \& Harlan, J.M. (1987) A monoclonal antibody to the membrane glycoprotein complex CD18 inhibits polymorphonuclear leukocyte accumulation and plasma leakage in vivo. Blood, 69, $338-340$.

3) Arnaout, M.A., Hakim, R.M., Todd, R.B., Dana, N. \& Colten, H.R. (1985) Increased expression of an adhesion-promoting surface glycoprotein in the granurocytopenia of hemodialysis. N. Engl. J. Med., 312, 457-462.

4) Barroso-Aranda, J., Schmid-Schonbein, Gw, Zweifach, B.W. \& Mathison, J.C. (1991) Polymorphonuclear neutrophil contribution to induced tolerance to bacterial lipopolysaccharide. Circ. Res., 69, 1196-1206.

5) Doerschuk, C.M. (1992) The role of CD18-mediated adhesion in neutrophil sequestration induced by infusion of activated plasma in rabbits. Am. J. Respir. Cell Mol. Biol., 7, 140-148.

6) Freyer, D.R. (1989) Surface Mol (CD11b/CD18) glycoprotein is up-modulated by neutrophils recruited to sites of inflammation in vivo. Inflammation, 13, 495-505.

7) Horgan, M.J., Wright, S.D. \& Malik, A.B. (1990) Antibody against leukocyte integrin (CD18) prevents reperfusion-induced lung vascular injury. Am. J. Physiol., 259, L315-L319.

8) Koike, K., Albertine, K.H. \& Staub, N.C. (1986) Intrathoracic sources of caudal mediastinal lymph node efferent lymph in sheep. J. Appl. Physiol., 60, 80-84.

9) Labeta, M.O., Durieux, J.J., Spagnoli, G., Fernandez, N., Wijdenes, J. \& Herrmann, R. (1993) CD14 and tolerance to lipopolysaccharide: Biochemical and functional analysis. Immunology, 80, 415-423.

10) Lundberg, C.A.D.W. (1990) Relation of the CD11/CD18 family of leukocyte antigens to the transient neutropenia caused by chemoattractants. Blood, 76, 12401245.

11) Mackensen, A., Galanos, C. \& Engelhardt, R. (1991) Modulating activity of interferon-gamma on endotoxin-induced cytokine production in cancer patients. Blood, 78, 3254-3258.

12) Mileski, W., Borgstrom, D., Lightfoot, E., Rothlein, R., Faanes, R., Lipsky, P. \& Baxter, C. (1992) Inhibition of leukocyte-endothelial adherence following thermal injury. J. Surg. Res., 52, 334-339.

13) Powe, J.E. (1982) Pulmonary accumulation of polymorphonuclear leukocytes in the adult respiratory distress syndrome. Crit. Care Med., 10, 712-718.

14) Schleiffenbaum, B., Moser, R., Patarroyo, M. \& Fehr, J. (1989) The cell surface glycoprotein Mac-1 (CD11b/CD18) mediates neutrophil adhesion and modulates degranulation independently of its quantitative cell surface expression. J. Immunol., 142, 3537-3545.

15) Staub, N., Bland, R., Brigham, K.L., Demiling, R., Erdman, J. \& Wolverton, W. (1975) Preparation of chronic lung lymph fistulas in sheep. J. Surg. Res., 19, 315320.

16) Staub, N.C., Schultz, E.L. \& Albertine, K.H. (1982) Leukocytes and pulmonary microvascular injury. Ann. N.Y. Acad. Sci., 384, 332-343. 
17) Thomas, J.R., Harlan, J.M., Rice, C.L. \& Winn, R.K. (1992) Role of leukocyte CD11/CD18 complex in endotoxic and septic shock in rabbits. J. Appl. Physiol., 73, 1510-1516.

18) Virca, G.D., Kim, S.Y., Glaser, K.B. \& Ulevitch, R.J. (1989) Lipopolysaccharide induces hyporesponsiveness to its own action in RAW 264.7 cells. J. Biol. Chem., 264, 21951-21956.

19) Warner, A.E. (1988) Pulmonary removal of circulating endotoxin results in acute lung injury in sheep. Lab. Invest., 59, 219-230. 